This is a pre-copyedited version of a contribution published in R. Haynes et al. (eds) Domain Decomposition Methods in Science and Engineering XXV. Lecture Notes in Computational Science and Engineering, vol 138 published by Springer Nature Switzerland AG. The definitive authenticated version is available online via https://1ink. springer. com/chapter/10.1007/978-3-030-56750-7_18.

\title{
Local spectra of adaptive domain decomposition methods
}

\author{
Alexander Heinlein ${ }^{1,2}$, Axel Klawonn ${ }^{1,2}$, and Martin J. Kühn ${ }^{3}$
}

\section{Introduction}

For second order elliptic partial differential equations, such as diffusion or elasticity, with arbitrary and high coefficient jumps, the convergence rate of domain decomposition methods with classical coarse spaces typically deteriorates. One remedy is the use of adaptive coarse spaces, which use eigenfunctions computed from local generalized eigenvalue problems to enrich the standard coarse space; see, e.g., $[19,6,5,4,22,23,3,16,17,14,7,8,24,1,20,2,13,21,10,9,11]$. This typically results in a condition number estimate of the form

$$
\kappa \leq C \text { tol } \quad \text { or } \quad \kappa \leq C \frac{1}{\text { tol }}
$$

of the preconditioned system, where $C$ is independent of the coefficient function and tol is a tolerance for the selection of the eigenfunctions.

Obviously, the robustness of the adaptive domain decomposition methods is therefore closely related to the choice of tol. Whereas for a pessimistic choice, i.e., tol $\approx 1$, the adaptive coarse space can resort to a direct solver, a very optimistic choice can lead to bad convergence behavior of the method.

In this article, we will compare the spectra of the generalized eigenvalue problems of several adaptive coarse spaces for overlapping as well as nonoverlapping domain decomposition methods. The spectra are of interest because they provide information for choosing an adequate tolerance splitting bad and good eigenmodes as well as about the resulting dimension of the adaptive coarse spaces. Therefore, we will consider certain representative examples of coefficient functions in two dimensions.

\footnotetext{
${ }^{1}$ Department of Mathematics and Computer Science, University of Cologne, Weyertal 86-90, 50931 Köln, Germany, e-mail: \\{alexander .heinlein, axel.klawonn $\backslash\} @ u n i-k o e l n . d e$ ${ }^{2}$ Center for Data and Simulation Science, University of Cologne, url: http://www.cds. uni-koeln.de

${ }^{3}$ CERFACS (Centre Européen de Recherche et de Formation Avancée en Calcul Scientifique), 42 Avenue Gaspard Coriolis, 31057 Toulouse Cedex 01, France; martin.kuehn@ cerfacs.fr.
} 
Note that we are not going to discuss other important properties of the adaptive coarse spaces considered here, such as

- condition number and iteration counts of the methods,

- costs for the computation of the eigenvalue problems and the coarse basis functions, respectively,

- necessary communication in a parallel implementation and the ratio of local and global work.

Thus, we do not claim to draw a general comparison of the different adaptive methods. We only want to discuss reasonable choices for the user-defined tolerance for different, exemplary coefficient distributions and the different types of eigenvalue problems. We hope that this gives some insight for further discussions.

Model problems and domain decomposition notation We consider the variational form of a second order elliptic partial differential equation, such as diffusion or elasticity, and denote the coefficient by $\rho \in \mathbb{R}^{+}$which is assumed to be constant on each finite element. In matrix form, the problem reads $A x=b$.

Now, let $\Omega$ be decomposed into nonoverlapping subdomains $\Omega_{1}, \ldots, \Omega_{N}$ and $\Gamma$ be the interface of this domain decomposition. We define corresponding subdomain stiffness matrices $A^{(i)}$ with Neumann boundary conditions on $\partial \Omega_{i}, i=1, \ldots, N$ and the block diagonal matrix $A_{N}:=\operatorname{blockdiag}_{i}\left(A^{(i)}\right)$ which is not assembled in the interface degrees of freedom. For an edge $\mathcal{E}$ or its closure $\overline{\mathcal{E}}$ shared by the subdomains $\Omega_{i}$ and $\Omega_{j}$, we obtain the matrix $A_{a}^{(i, j)}$ by assembly of the degrees of freedom on $\mathcal{E}$ or $\overline{\mathcal{E}}$, respectively, in the matrix $A_{n a}^{(i, j)}:=\operatorname{blockdiag}\left(A^{(i)}, A^{(j)}\right)$.

The Schur complements with respect to $\mathcal{Z}=\mathcal{E}, \mathcal{Z}=\overline{\mathcal{E}}$, or any other $\mathcal{Z} \subset \Gamma$ are obtained from $A_{n a}^{(i, j)}$ or $A_{a}^{(i, j)}$ by elimination of all remaining local degrees of freedom $Z^{C}$ :

$$
S_{*, \mathcal{Z}}^{(i, j)}:=A_{*, \mathcal{Z} Z}^{(i, j)}-A_{*, \mathcal{Z} \mathcal{Z}^{C}}^{(i, j)}\left(A_{*, Z^{C} \mathcal{Z}^{C}}^{(i, j)}\right)^{-1} A_{*, Z^{C} \mathcal{Z}}^{(i, j)}
$$

with $* \in\{a, n a\}$. We also need $S_{\mathcal{Z}}^{(i)}:=A_{\mathcal{Z} \mathcal{Z}^{(i)}}-A_{\mathcal{Z} \mathcal{Z}^{C}}^{(i)}\left(A_{\mathcal{Z}^{C} \mathcal{Z}^{C}}^{(i)}\right)^{-1} A_{\mathcal{Z}^{C}}^{(i)} \mathcal{Z}^{\text {. }}$

In addition to that, let the matrices $A_{\mathcal{E}}$ and $M_{\mathcal{E}}$ be matrix discretizations of the onedimensional bilinear forms $a_{\mathcal{E}}(u, v):=\int_{\mathcal{E}} \rho_{\mathcal{E}, \max } D_{x^{t}} u D_{x^{t}} v d x$ and $b_{\mathcal{E}}(u, v):=$ $h^{-1} \sum_{x_{k} \in \mathcal{E}} \beta_{k} u\left(x_{k}\right) v\left(x_{k}\right)$ with $\beta_{k}:=\sum_{\left\{t \in \tau_{h}: k \in \operatorname{dof}(t)\right\}} \rho_{t}$. Here, $\rho_{t}$ is the constant coefficient on the element $t \in \tau_{h}$ and $\rho_{\mathcal{E}, \max }(x):=\max \left\{\lim _{y_{i} \in \Omega_{i} \rightarrow x} \rho\left(y_{i}\right), \lim _{y_{j} \in \Omega_{j} \rightarrow x} \rho\left(y_{j}\right)\right\}$. $D_{x^{t}}$ denotes the tangent derivative with respect to the edge $e_{i j}$, and the $x_{k}$ correspond to the finite element nodes on the edge. Consequently, $A_{\mathcal{E}}$ and $M_{\mathcal{E}}$ are the stiffness matrix and a scaled lumped mass matrix on the edge $\mathcal{E}$.

\section{Various adaptive coarse spaces in domain decomposition}

Overlapping Schwarz methods We extend the nonoverlapping subdomains to overlapping subdomains $\Omega_{1}^{\prime}, \ldots, \Omega_{N}^{\prime}$ and consider two-level overlapping Schwarz methods of the form 


$$
M_{O S-2}^{-1}=\Phi A_{0}^{-1} \Phi^{T}+\sum_{i=1}^{N} R_{i}^{T} A_{i}^{-1} R_{i}
$$

with overlapping matrices $A_{i}=R_{i} A R_{i}^{T}, i=1, \ldots, N$, where $R_{i}$ is the restriction matrix to the overlapping subdomain $\Omega_{i}^{\prime}$, and the coarse matrix $A_{0}=\Phi^{T} A \Phi$. Here, the columns of $\Phi$ are the coarse basis functions. We consider three different adaptive coarse spaces for overlapping Schwarz methods, i.e., the Spectral Harmonically Enriched Multiscale (SHEM) [7], the Overlapping Schwarz Approximate Component Mode Synthesis (OS-ACMS) [10], and the Adaptive Generalized Dryja-SmithWidlund (AGDSW) $[9,11]$ coarse spaces.

In all these approaches, the coarse space consists of vertex- and edge-based energy-minimizing basis functions, i.e., the interior values $\Phi_{I}$ are given by $\Phi_{I}:=$ $-A_{I I}^{-1} A_{I \Gamma} \Phi_{\Gamma}$ for given interface values $\Phi_{\Gamma}$. The vertex-based basis functions are nodal basis functions of Multiscale Finite Element Method (MsFEM) [12] type with different choices of edge values; cf. [7, 10, 9, 11]. The edge-based basis functions are energy-minimizing extensions of the solutions of generalized eigenvalue problems corresponding to the edges of the nonoverlapping domain decomposition.

For an edge $\mathcal{E}$ of the nonoverlapping domain decomposition, we consider the following edge eigenvalue problems.

(Ov1) SHEM coarse space [7]: find $\left(\tau_{\mathcal{E}}, \mu_{\mathcal{E}}\right) \in V_{0}^{h}(\mathcal{E}) \times \mathbb{R}$ s.t.

$$
\theta^{T} A_{\mathcal{E}} \tau_{\mathcal{E}}=\mu_{\mathcal{E}}^{-1} \theta^{T} M_{\mathcal{E}} \tau_{\mathcal{E}} \quad \forall \theta \in V_{0}^{h}(\mathcal{E}) .
$$

(Ov2) OS-ACMS coarse space [10]: find $\left(\tau_{\mathcal{E}}, \mu_{\mathcal{E}}\right) \in V_{0}^{h}(\mathcal{E}) \times \mathbb{R}$ s. t.

$$
\theta^{T} S_{\overline{\mathcal{E}}}^{(i, j)} \tau_{\mathcal{E}}=\mu_{\mathcal{E}}^{-1} \theta^{T} A_{\overline{\mathcal{E}} \overline{\mathcal{E}}} \tau_{\mathcal{E}} \quad \forall \theta \in V_{0}^{h}(\mathcal{E}) .
$$

(Ov3) AGDSW coarse space [9, 11]: find $\left(\tau_{\mathcal{E}}, \mu_{\mathcal{E}}\right) \in V_{0}^{h}(\mathcal{E}) \times \mathbb{R}$ s.t.

$$
\theta^{T} S_{\mathcal{E}}^{(i, j)} \tau_{\mathcal{E}}=\mu_{\mathcal{E}}^{-1} \theta^{T} A_{\mathcal{E} \mathcal{E}} \tau_{\mathcal{E}} \quad \forall \theta \in V_{0}^{h}(\mathcal{E}) .
$$

Let the reciprocal eigenvalues $\mu_{\mathcal{E}}$ be ordered nondescendingly. Then, we select eigenpairs with $\mu_{\mathcal{E}}>$ tol to obtain a condition number estimate of the form $\kappa\left(M_{O S 2}^{-1} A\right) \leq C$ tol that is independent of the coefficient function $\rho$. Note that we use the reciprocal eigenvalue only for comparison with the adaptive coarse spaces for nonoverlapping domain decomposition methods. For the AGDSW coarse space, the matrix on the left hand side is singular. Therefore, we obtain infinity reciprocal eigenvalues in our numerical results.

Nonoverlapping methods In the nonoverlapping domain decomposition methods FETI-1 and FETI-DP, we use the block diagonal matrix $A_{N}$ and introduce a jump operator $B$ for the interface with $B:=\left(B_{1}, \ldots, B_{N}\right), u=\left(u_{1}^{T}, \ldots, u_{N}^{T}\right)^{T}$, and $u_{i}: \Omega_{i} \rightarrow \mathbb{R}, i=1, \ldots, N$ such that $B u=0$ if and only if $u$ is continuous across the interface. The FETI master system is given by

$$
\left[\begin{array}{cc}
A_{N} & B^{T} \\
B & 0
\end{array}\right]\left[\begin{array}{l}
u \\
\lambda
\end{array}\right]=\left[\begin{array}{l}
f \\
0
\end{array}\right] .
$$


In FETI-1, the null space of $A_{N}$ is handled by a projection $P$ such that we solve the following system reduced to the Lagrange multipliers and preconditioned by the nonadaptive, projected Dirichlet preconditioner $P M_{D}^{-1} P^{T}$

$$
P M_{D}^{-1} P^{T} B A_{N}^{+} B^{T} P^{T} \lambda=P M_{D}^{-1} P^{T} d
$$

with corresponding right hand side $P M_{D}^{-1} P^{T} d$. We have $M_{D}^{-1}=B_{D}$ blockdiag $\left(S_{\Gamma_{i}}^{(i)}\right) B_{D}^{T}$, where $B_{D}$ is a scaled variant of $B$. In FETI-DP, we subassemble $A_{N}$ in a selected number of degrees of freedom on the interface, e.g., all vertices, and denote the resulting nonsingular matrix by $\widetilde{A}_{N}$. In the nonadaptive case, we then solve the preconditioned system

$$
M_{D}^{-1} B \widetilde{A}_{N}^{-1} B^{T} \lambda=M_{D}^{-1} \widetilde{d} .
$$

Adaptive constraints can then be enforced by, e.g., a second projection $P_{0}$ (see [23] for FETI-1 or [17, 14] for FETI-DP) or via a generalized transformation-of-basis approach; see [15]. In FETI-1/-DP and BDD(C) methods, the operator $P_{D}=B_{D}^{T} B$ is used for proving condition number bounds and thus also appears in some generalized eigenvalue problems.

In this paper, we consider the the GenEO eigenvalue problems for FETI-1 (or BDD methods); see [23]; which were first introduced for overlapping Schwarz methods; see [22]. A $P_{D}$-based estimate based coarse space was motivated in [19]. There, $P_{D}$ was localized to $P_{D, \mathcal{E}}$ by extracting from $B$ and $B_{D}$ the rows only considering the jumps on the corresponding edge (in 2D). A condition number bound for the 2D case was proven in [17]. The method was extended to a robust three dimensional version in [14]. We present results with $\rho$-scaling as (NOv2a) and deluxe-scaling as (NOv2b). Another $P_{D}$-based coarse space was proposed by [3] for BDDC with deluxe-scaling. In the eigenvalue problems, the matrix operator $A: B=A(A+B)^{+} B$ is used and the cutoff of the interface Schur complement at the edge $S_{\Gamma_{i \mid \varepsilon}}^{(i)}$ is used on the right hand side. The energy comparison was generalized to arbitrary scaling matrices $D^{(i)}$ in [17]. Extensions of this method to three dimensions were considered, e.g., in $[24,1,20,2,13]$. We present results for $\rho$-scaling as (NOv3a) and deluxe-scaling as (NOv3b).

(NOv1) GenEO coarse space (FETI-1/BDD) [23]: find $\left(\tau_{\Gamma_{i}}, \mu_{\Gamma_{i}}\right) \in V^{h}\left(\Gamma_{i}\right) \times \mathbb{R}$ s.t.

$$
\theta^{T} S_{\Gamma_{i}}^{(i)} \tau_{\Gamma_{i}}=\mu_{\Gamma_{i}}^{-1} \theta^{T}\left(B_{i}^{T} M_{D}^{-1} B_{i}\right) \tau_{\Gamma_{i}} \quad \forall \theta \in V^{h}\left(\Gamma_{i}\right) .
$$

(NOv2) $P_{D}$-based coarse space no. 1 (FETI-DP/BDDC) [19]: find $\left(\tau_{\Gamma_{i}}, \mu_{\Gamma_{i}}\right) \in$ $\left(\operatorname{ker} S_{n a, \Gamma_{i j}}^{(i, j)}\right)^{\perp} \times \mathbb{R}$ s.t.

$$
\theta^{T} P_{D, \mathcal{E}}^{T} S_{n a, \Gamma_{i j}}^{(i, j)} P_{D, \mathcal{E}} \tau_{\Gamma_{i j}}=\mu_{\Gamma_{i j}} \theta^{T} S_{n a, \Gamma_{i j}}^{(i, j)} \tau_{\Gamma_{i j}} \quad \forall \theta \in\left(\operatorname{ker} S_{n a, \Gamma_{i j}}^{(i, j)}\right)^{\perp} .
$$

(NOv3) $P_{D}$-based coarse space no. 2 (FETI-DP/BDDC) [3]: find $\left(\tau_{\mathcal{E}}, \mu_{\mathcal{E}}\right) \in$ $V_{0}^{h}(\mathcal{E}) \times \mathbb{R}$ s.t.

$$
\theta^{T} S_{\mathcal{E}}^{(i)}: S_{\mathcal{E}}^{(j)} \tau_{\mathcal{E}}=\mu_{\mathcal{E}} \theta^{T}\left(D_{\mathcal{E}}^{(j), T} S_{\Gamma_{\mid \mathcal{E}}}^{(i)} D_{\mathcal{E}}^{(j)}+D_{\mathcal{E}}^{(i), T} S_{\Gamma_{\mid \mathcal{E}}}^{(j)} D_{\mathcal{E}}^{(i)}\right) \tau_{\mathcal{E}} \quad \forall \theta \in V_{0}^{h}(\mathcal{E})
$$


Let the (reciprocal) eigenvalues be ordered nondescendingly. Then, we select eigenpairs with $\mu_{\Gamma_{i}}^{-1}, \mu_{\Gamma_{i j}}$, or $\mu_{\mathcal{E}}$ greater than tol. For the (NOv1) and the (NOv3) eigenvalue problems, the matrix on the left hand side is singular, therefore, we obtain infinity (reciprocal) eigenvalues in our numerical results. For (NOv1), note that the authors of [23] do not incorporate the eigenvectors corresponding to zero eigenvalues into the coarse space. With all three eigenvalue problems (NOv1)-(NOv3), we then obtain adaptive methods with a condition number bound $\kappa \leq C$ tol that is independent of the coefficient function $\rho$.

\section{Numerical results}

In this section, we present results for a diffusion problem on $\Omega=(0,1)^{2}$ decomposed into nine subdomains. We used a rectangular domain decomposition and slightly curved edges for the subdomain in the center to prevent the appearance of symmetric effects. We set homogeneous Dirichlet boundary conditions for the edge with $x=0$ and homogeneous Neumann boundary conditions elsewhere.

The local spectra of the different adaptive coarse spaces for eight different coefficient distributions are shown in Figures 1 and 2. The critical eigenvalues and reciprocal eigenvalues, respectively, are displayed above the spectral gap, which is hatched in gray. They are plotted side by side if they are close to each other. A wide spectral gap simplifies the choice of an appropriate tolerance tol. In addition to that, the number of critical eigenvalues is related to the dimension of the coarse space. Note that the condition number estimate (1) guarantees fast convergence of all different approaches for arbitrary coefficient distributions if a suitable tolerance is chosen. However, as can be observed from our results, there are significant differences in the width of the spectral gap and the number of critical eigenvalues for the depicted model problems.

The use of harmonic extensions in the eigenvalue problems of the OS-ACMS coarse space can reduce the number of bad eigenmodes compared to the cheaper one-dimensional integrals in the related SHEM coarse space. A similar behavior can be observed for the expensive deluxe-scaling compared to the cheaper $\rho$-scaling for the $P_{D}$-based approaches for FETI-DP/BDDC. For several coefficient distributions, the width of the spectral gap is larger than two orders of magnitude for all approaches, whereas it is quite small, e.g., for channel-type coefficient distributions.

Note that the plots in Figures 1 and 2 contain much more information, which we cannot discuss here due to lack of space. We hope that the results presented here give some insight for further investigations. Further investigations in three dimensions are also of high interest. This is however out of the scope of this paper. A small comparison between the 3D version algorithms of columns (Nov2a) and (Nov2b) can be found in [18, Sec. 6.5.3]. For overlapping Schwarz methods, a comparison between different 3D approaches including (Ov3) can be found in [11]. 

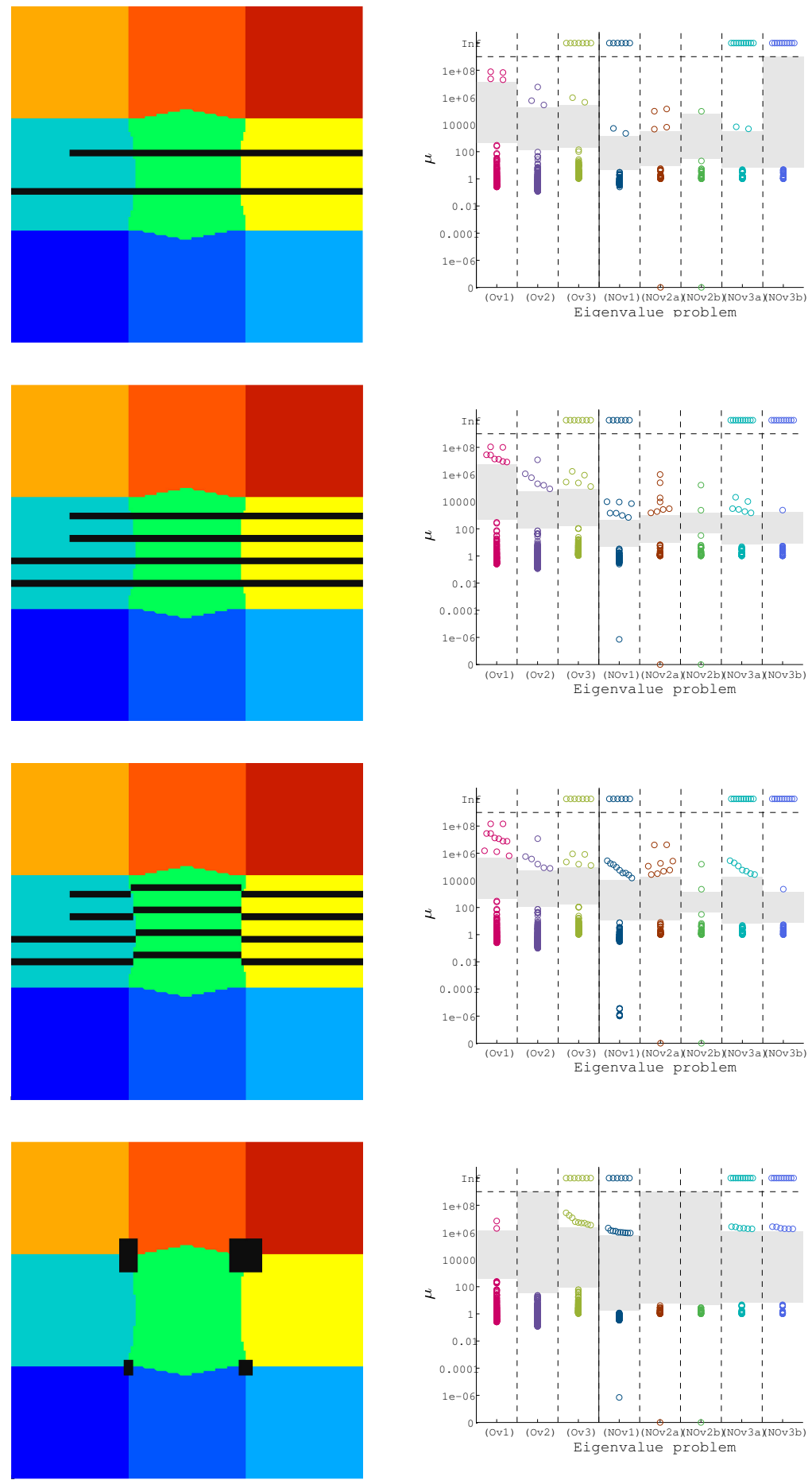

Fig. 1 For each exemplary coefficient distribution, domain decomposition and coefficient distribution is shown on the left hand side. Large coefficients with $\rho=1 e 6$ are shown in black (low coefficients with $\rho=1$ are not shown). The different subdomains are shown in different colors on a layer underneath the large coefficients. The corresponding (reciprocal) eigenvalues $\mu$ are shown on the right hand side. Large values (greater 500) are distributed horizontally within the columns to visualize their number. The gap between good and bad eigenmodes is shown in gray. 

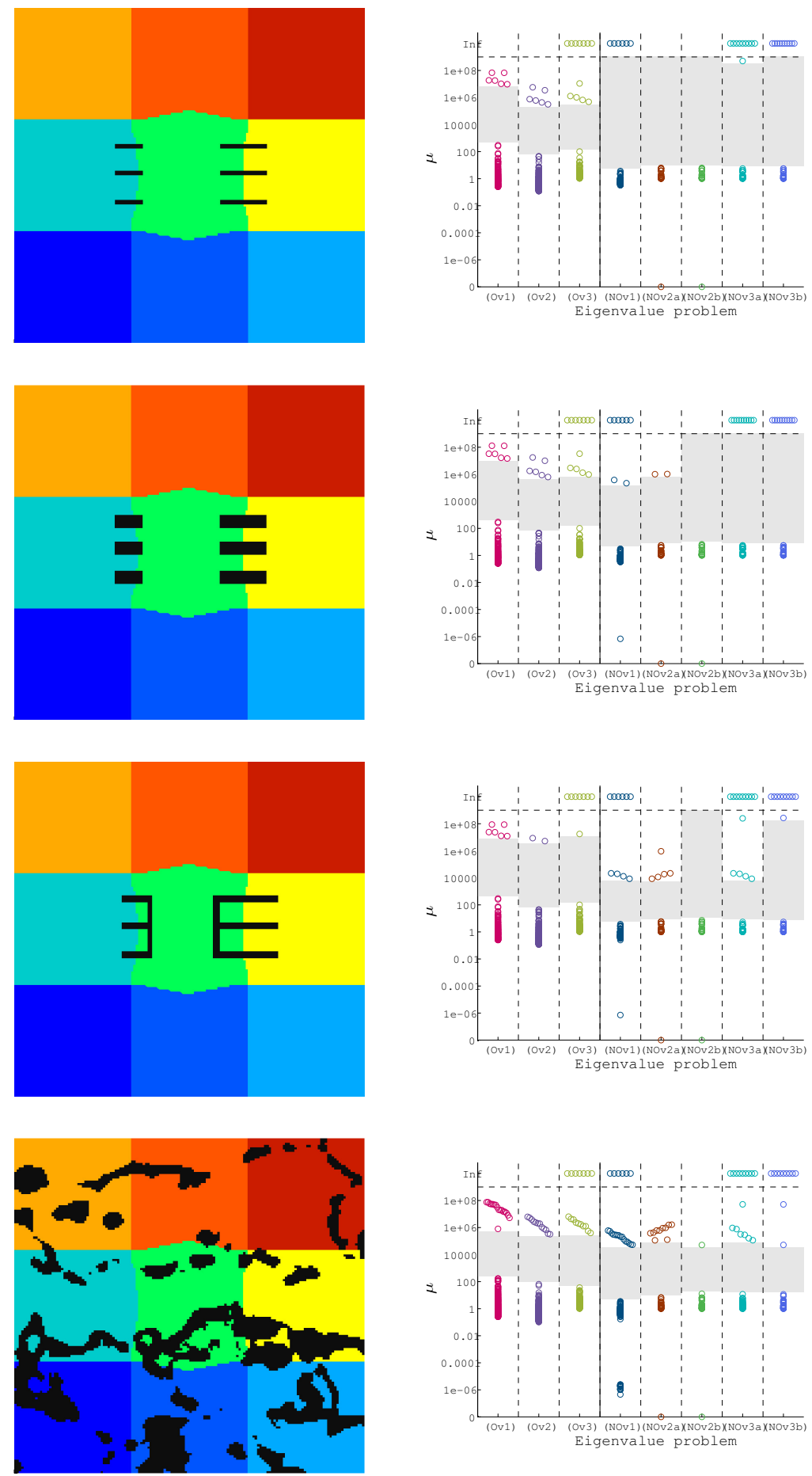

Fig. 2 For each exemplary coefficient distribution, domain decomposition and coefficient distribution is shown on the left hand side. Bottom image: coefficient function generated from the microstructure of a dual-phase steel; courtesy of Jörg Schröder, University of Duisburg-Essen, Germany, originating from a cooperation with ThyssenKruppSteel. Large coefficients with $\rho=1 e 6$ are shown in black (low coefficients with $\rho=1$ are not shown). The different subdomains are shown in different colors on a layer underneath the large coefficients. The corresponding (reciprocal) eigenvalues $\mu$ are shown on the right hand side. Large values (greater 500) are distributed horizontally within the columns to visualize their number. The gap between good and bad eigenmodes is shown in gray. 


\section{References}

1. L. Beirão da Veiga, L. F. Pavarino, S. Scacchi, O. B. Widlund, and S. Zampini. Adaptive selection of primal constraints for isogeometric BDDC deluxe preconditioners. SIAM J. Sci. Comput., 39(1):A281-A302, 2017.

2. J. G. Calvo and O. B. Widlund. An adaptive choice of primal constraints for BDDC domain decomposition algorithms. Electronic Transactions on Numerical Analysis, 45:524-544, 2016.

3. C. Dohrmann and C. Pechstein. In C. Pechstein, Modern domain decomposition solvers - BDDC, deluxe scaling, and an algebraic approach. Slides to a talk at NuMa Seminar, JKU Linz, December 10th, 2013, http://people.ricam.oeaw.ac.at/c.pechstein/ pechstein-bddc2013.pdf, 2013.

4. V. Dolean, F. Nataf, R. Scheichl, and N. Spillane. Analysis of a two-level Schwarz method with coarse spaces based on local Dirichlet-to-Neumann maps. Comput. Methods Appl. Math., 12(4):391-414, 2012.

5. Y. Efendiev, J. Galvis, R. Lazarov, and J. Willems. Robust domain decomposition preconditioners for abstract symmetric positive definite bilinear forms. ESAIM: Mathematical Modelling and Numerical Analysis, 46(05):1175-1199, 2012.

6. J. Galvis and Y. Efendiev. Domain decomposition preconditioners for multiscale flows in high-contrast media. Multiscale Modeling \& Simulation, 8(4):1461-1483, 2010.

7. M. J. Gander, A. Loneland, and T. Rahman. Analysis of a new harmonically enriched multiscale coarse space for domain decomposition methods. Technical report, arxiv.org, 2015.

8. R. Haferssas, P. Jolivet, and F. Nataf. A robust coarse space for optimized Schwarz methods: SORAS-GenEO-2. C. R. Math. Acad. Sci. Paris, 353(10):959-963, 2015.

9. A. Heinlein, A. Klawonn, J. Knepper, and O. Rheinbach. An adaptive GDSW coarse space for two-level overlapping Schwarz methods in two dimensions. In Domain Decomposition Methods in Science and Engineering XXIV, volume 125 of LNCSE. Springer, 2018.

10. A. Heinlein, A. Klawonn, J. Knepper, and O. Rheinbach. Multiscale coarse spaces for overlapping Schwarz methods based on the ACMS space in 2D. ETNA, 48:156-182, 2018.

11. A. Heinlein, J. Knepper, A. Klawonn, and O. Rheinbach. Adaptive GDSW coarse spaces for overlapping schwarz methods in three dimensions. Technical report, 2018. Submitted to SISC.

12. T. Y. Hou and X.-H. Wu. A multiscale finite element method for elliptic problems in composite materials and porous media. Journal of Computational Physics, 134(1):169 - 189, 1997.

13. H. H. Kim, E. Chung, and J. Wang. BDDC and FETI-DP preconditioners with adaptive coarse spaces for three-dimensional elliptic problems with oscillatory and high contrast coefficients. J. Comput. Phys., 349:191-214, 2017.

14. A. Klawonn, M. Kühn, and O. Rheinbach. Adaptive coarse spaces for FETI-DP in three dimensions. SIAM J. Sci. Comput., 38(5):A2880-A2911, 2016.

15. A. Klawonn, M. Kühn, and O. Rheinbach. Adaptive FETI-DP and BDDC methods with a generalized transformation of basis for heterogeneous problems. Electronic Transactions on Numerical Analysis (ETNA), 49:1-27, 2018.

16. A. Klawonn, P. Radtke, and O. Rheinbach. FETI-DP methods with an adaptive coarse space. SIAM J. Numer. Anal., 53(1):297-320, 2015.

17. A. Klawonn, P. Radtke, and O. Rheinbach. A comparison of adaptive coarse spaces for iterative substructuring in two dimensions. Electron. Trans. Numer. Anal., 45:75-106, 2016.

18. M. J. Kühn. Adaptive FETI-DP and BDDC methods for highly heterogeneous elliptic finite element problems in three dimensions. PhD thesis, Universität zu Köln, 2018.

19. J. Mandel and B. Sousedík. Adaptive selection of face coarse degrees of freedom in the BDDC and the FETI-DP iterative substructuring methods. Comput. Methods Appl. Mech. Engrg., 196(8):1389-1399, 2007.

20. D. Oh, O. B. Widlund, S. Zampini, and C. R. Dohrmann. BDDC Algorithms with deluxe scaling and adaptive selection of primal constraints for Raviart-Thomas vector fields. Math. Comp., 87(310):659-692, 2018.

21. C. Pechstein and C. R. Dohrmann. A unified framework for adaptive BDDC. Electron. Trans. Numer. Anal., 46:273-336, 2017. 
22. N. Spillane, V. Dolean, P. Hauret, F. Nataf, C. Pechstein, and Robert Scheichl. Abstract robust coarse spaces for systems of PDEs via generalized eigenproblems in the overlaps. Numer. Math., 126(4):741-770, 2014.

23. N. Spillane and D. J. Rixen. Automatic spectral coarse spaces for robust finite element tearing and interconnecting and balanced domain decomposition algorithms. Internat. J. Numer. Methods Engrg., 95(11):953-990, 2013.

24. S. Zampini. PCBDDC: a class of robust dual-primal methods in PETSc. SIAM J. Sci. Comput., 38(5):S282-S306, 2016. 